\title{
High serum levels of YKL-40 in patients with systemic sclerosis are associated with pulmonary involvement
}

\author{
C Nordenbæk', JS Johansen ${ }^{1,2}$, P Halberg ${ }^{1}$, A Wiik ${ }^{3}$, C Garbarsch ${ }^{4}$, S Ullman ${ }^{5}$, PA Price ${ }^{6}$, S Jacobsen ${ }^{1,7}$ \\ ${ }^{1}$ Department of Rheumatology, Hvidovre Hospital, ${ }^{2}$ Department of Rheumatology, Herlev Hospital, ${ }^{3}$ Department of Autoimmunology, \\ Statens Serum Institute, Copenhagen, ${ }^{4}$ Institute of Medical Anatomy, The Panum Institute, Copenhagen, ${ }^{5}$ Department of Dermatology, \\ Bispebjerg Hospital, Copenhagen, Denmark, ${ }^{6}$ Department of Biology, University of California San Diego, La Jolla, CA, USA, and \\ ${ }^{7}$ Department of Rheumatology, Rigshospitalet, Copenhagen, Denmark
}

Objectives: YKL-40, a growth factor of connective tissue cells, is elevated in sera from patients with diseases characterized by inflammation, tissue remodelling, or fibrosis. The aim of the study was to determine serum YKL-40 levels in patients with systemic sclerosis (SSc) and to explore any possible clinical and prognostic associations.

Methods: YKL-40 was measured in sera from 88 patients with SSc (26 with diffuse and 62 with limited skin involvement) and in sera from 88 matched healthy controls. Immunohistochemical staining for YKL-40 antigen was performed in a biopsy from a patient with pulmonary SSc.

Results: Serum YKL-40 levels of the SSc patients were significantly higher than those of the controls $(\mathrm{p}<0.00001)$. Patients with pulmonary fibrosis by chest X-ray, obstructive ventilatory pattern, reduced diffusing capacity (DLco), and digital joint deformity due to skin retraction had significantly higher serum YKL-40 compared with patients without these findings. Patients with elevated serum YKL-40 had shorter survival times than patients with normal serum YKL-40 $(\mathrm{p}=0.0005)$, although this was not independent of age and pulmonary function. YKL-40 protein expression was found in inflammatory cells in fibrosing pulmonary tissue from a patient with SSc.

Conclusions: Serum YKL-40 is elevated in patients with SSc with pulmonary involvement.

YKL-40, also known as human cartilage glycoprotein39 (1), is a member of the 'mammalian chitinase-like proteins'. The gene (CHI3LI) for YKL-40 (2) and its crystallographic structure (3) are known, but the site and mode of binding to cell surface receptors have not yet been described. YKL-40 is a heparin and chitinbinding lectin (4) without chitinase activity $(1,4)$. It is produced by macrophages $(4,5)$, neutrophils $(6)$, synovial cells, chondrocytes from arthritic joints (1), differentiating smooth muscle cells, hepatic stellate cells, and cancer cells. The precise biological function of YKL-40 is unknown, but it increases growth rates of fibroblastic cell lines derived from human osteoarthritic synovium, foetal lung and adult skin, and works synergistically with insulin-like growth factor (IGF)-1 in stimulating the growth of fibroblasts (7). YKL-40 also increases the number of chondrocytes and synovial cells and proteoglycan synthesis (8). Furthermore, YKL-40 is an adhesion and migration

Søren Jacobsen, Department of Rheumatology, Rigshospitalet, Blegdamsvej 9, DK-2100 Copenhagen, Denmark.

E-mail: sj@dadlnet.dk

Received 29 June 2004

Accepted 7 January 2004 factor for vascular smooth muscle cells (9) and umbilical vein endothelial cells and modulates vascular endothelial cell morphology by promoting the formation of branching tubules. This indicates that YKL-40 may function in angiogenesis. Besides having biological effects, YKL-40 has been shown to be an autoantigen in rheumatoid arthritis.

Elevated serum YKL-40 levels are found in patients with a variety of diseases characterized by inflammation, remodelling of the extracellular matrix, and development of fibrosis, such as rheumatoid arthritis, osteoarthritis, giant cell arteritis, pneumonia, liver fibrosis, and different types of solid tumours $(10,11)$. The serum YKL-40 levels vary according to the disease activity of the patients and elevated serum YKL-40 is associated with a poor prognosis.

Systemic sclerosis (SSc) is an autoimmune disease characterized by initial inflammation followed by fibrotic changes in the skin, blood vessels, and several other organs. The pathogenesis of SSc is unclear. However, various growth factors seem to be involved in the fibrotic processes (12). Elevated serum YKL-40 is found in approximately $30 \%$ of patients with SSc and particularly in those with joint involvement (13). The aim of the present study was to assess 
whether serum YKL-40 in patients with SSc has clinical and prognostic associations.

\section{Material and methods}

\section{Patients}

Eighty-eight patients (median age 58 years, range 20-82) who fulfilled the American College of Rheumatology (ACR) preliminary criteria for the classification of SSc were included, and were part of a longitudinally followed population (14). The patients were selected according to the availability of serum samples collected during the follow-up. The median duration of cutaneous sclerosis was 7.2 years (range $0-39$ ) and the median duration of follow-up after the time of blood sampling was 3.2 years (range $0-17$ ). Skin involvement was diffuse in $26(30 \%)$ patients and limited in $62(70 \%)$. Fifty-five patients were (or had been) treated with penicillamine $(300-1200 \mathrm{mg}$ daily, median $750 \mathrm{mg}$ ). Skin involvement was classified according to the maximum cutaneous involvement by means of a two-subset model (limited and diffuse involvement; dividing lines: elbows and knees). Demographic data, signs, and symptoms (i.e. gender, age, duration of cutaneous involvement, years of follow-up after blood sampling, digital pitting scars, subcutaneous calcinosis, distal osteolysis, chronic polyarthritis, SSc-related deformities of the finger joints (due to retraction of skin and tendons), and the presence of anti-centromere and anti-Scl70 antibodies, pulmonary fibrosis) were registered at the time of blood sampling: Vital status was obtained from the Danish Central Person Register. Causes of death were based on information obtained from hospital charts, autopsy reports, and death certificates.

\section{Pulmonary imaging and function tests}

At inclusion, the patients had pulmonary function tests (PFTs) and routine chest X-ray examinations. The largest of three technically acceptable efforts was used to determine the forced expiratory volume during the first second (FEV1), inspiratory vital capacity (VC), and the ratio of FEV1/VC. The diffusing capacity of CO (DLco) was determined using a single breath helium, CO-dilution technique. The PFT results were transformed to standardized values calculated as percentages of the predicted values with respect to sex, age, and height. Three subsets of patients were delineated: (i) restrictive ventilatory pattern ( $\mathrm{VC} \leqslant 80 \%$ and $\mathrm{FEV} 1 / \mathrm{VC} \geqslant 70 \%$ ), (ii) obstructive ventilatory pattern $(\mathrm{FEV} 1 / \mathrm{VC}<70 \%)$, and (iii) DLco reduced to less than $70 \%$ of the expected values. Twenty-seven patients $(31 \%)$ had radiographically demonstrated pulmonary fibrosis. The ventilatory pattern was restrictive in $17(19 \%)$ patients, obstructive in $11(13 \%)$, and low DLco was found in $39(44 \%)$. In patients with pulmonary fibrosis the median values of $\mathrm{VC}$ and DLco were $76 \%$ and $56 \%$, respectively, of the expected value.

\section{Serological methods}

Serum YKL-40 was determined by an in-house radioimmunoassay (RIA). An age- and sex-matched control population consisting of 70 women and 18 men (median age 58 years, range 21-79) was used to determine the cut-off limit of normal versus elevated serum YKL-40 (i.e. the 95th confidence limit of serum YKL-40 in healthy subjects $=275 \mu \mathrm{g} / \mathrm{L}$ ).

\section{Statistical analysis}

Statistical analyses were performed by means of SPSS for Windows version 10 (SPSS Inc., Chicago, USA) and included the Mann-Whitney rank test, Fisher's exact test, stepwise conditional logistic regression analysis, and survival analyses by means of KaplanMeier plotting, log-rank statistics, and Cox regression analysis. Statistical significance was set at $\mathrm{p}<0.05$.

\section{Results}

Serum YKL-40 was higher in the patients (median $161 \mu \mathrm{g} / \mathrm{L}$, quartiles $112-284 \mu \mathrm{g} / \mathrm{L}$, 95 th percentile 602 , $\mathrm{p}<0.00001$ ) than in the controls (median $104 \mu \mathrm{g} / \mathrm{L}$, quartiles $89-149 \mu \mathrm{g} / \mathrm{L}, 95$ th percentile 275 ). Twentyseven per cent had elevated serum YKL-40 (i.e. $>$ the 95th percentile of the controls). Anticentromere antibodies (ACA) and antitopoisomerase-I antibodies (ATA) were found in 34 and 11 patients, respectively, and did not correlate with serum YKL-40.

Table 1 shows serum YKL-40 levels in relation to demographic and clinical features. Serum YKL-40 was significantly elevated in patients with pulmonary fibrosis, obstructive ventilatory pattern, SSc joint deformity, and old age ( $>60$ years). Table 2 shows the demographic and clinical features of the $88 \mathrm{SSc}$ patients stratified according to elevated or normal serum YKL-40. Elevated serum YKL-40 was significantly related to old age, obstructive ventilatory pattern, decreased vital capacity, and diffusing capacity. Elevated serum YKL-40 was not associated with restrictive ventilatory pattern. To evaluate independent contributions to elevated serum YKL-40, a logistic stepwise regression analysis of serum YKL-40 using the factors of Table 2 as independent variables was performed. The factors independently related to elevated serum YKL-40 included decreased values of DLco $(p=0.005)$ and decreased values of $\mathrm{FEV}_{1} / \mathrm{VC}(\mathrm{p}=0.05)$. 
Table 1. Serum concentrations of YKL-40 in 88 patients with systemic sclerosis according to the presence of selected demographic, clinical and serological features.

\begin{tabular}{|c|c|c|c|}
\hline & \multicolumn{2}{|c|}{ Serum YKL-40 ( $\mu \mathrm{g} / \mathrm{L})$} & \multirow[b]{2}{*}{$p^{*}$} \\
\hline & Feature present & Feature absent & \\
\hline Male sex $(n=18)$ & $224(108-312)$ & $156(116-312)$ & 0.38 \\
\hline Age $>60$ years $(n=38)$ & $220(142-352)$ & $136(96-224)$ & 0.004 \\
\hline Diffuse SSc $(n=26)$ & $155(136-284)$ & $170(108-284)$ & 0.95 \\
\hline Pitting scars $(n=59)$ & $188(128-288)$ & $136(96-248)$ & 0.19 \\
\hline Calcinosis $(n=41)$ & $188(136-304)$ & $146(96-276)$ & 0.07 \\
\hline Distal osteolysis ( $n=19)$ & $154(100-276)$ & $162(100-276)$ & 0.75 \\
\hline Arthritis $(n=7)$ & $276(156-312)$ & $156(108-272)$ & 0.25 \\
\hline SSc joint deformity $(n=61)$ & $194(136-288)$ & $128(80-224)$ & 0.03 \\
\hline Pulmonary fibrosis $(n=27)$ & $222(136-288)$ & $152(96-248)$ & 0.03 \\
\hline Isolated reduced DLco $(n=37)$ & $152(108-244)$ & $183(116-288)$ & 0.62 \\
\hline Restrictive pattern $(n=17)$ & $222(136-288)$ & $156(108-272)$ & 0.36 \\
\hline Obstructive pattern $(n=11)$ & $284(208-360)$ & $154(108-248)$ & 0.02 \\
\hline Smoking $(n=46)$ & $162(120-284)$ & $161(108-276)$ & 0.65 \\
\hline Anti-centromere antibody $(n=34)$ & $158(96-284)$ & $167(126-288)$ & 0.81 \\
\hline Anti-topoisomerase antibody $(n=11)$ & $154(80-288)$ & $162(116-284)$ & 0.77 \\
\hline
\end{tabular}

Values are medians (quartiles). *Mann-Whitney test.

Table 2. Demographic and clinical features stratified according to serum levels of YKL-40 in 88 patients with systemic sclerosis.

\begin{tabular}{|c|c|c|c|}
\hline & $\begin{array}{l}\text { High serum YKL-40 (>275 } \mu \mathrm{g} / \mathrm{L}) \\
\qquad \mathrm{n}=24\end{array}$ & $\begin{array}{l}\text { Normal serum } Y K L-40(\leqslant 275 \mu \mathrm{g} / \mathrm{L}) \text {, } \\
\mathrm{n}=64\end{array}$ & \\
\hline Binary variables & No. $(\%)$ & No. $(\%)$ & $p^{*}$ \\
\hline Male sex & $8(33)$ & $10(16)$ & 0.08 \\
\hline Smoking & $11(46)$ & $31(48)$ & 0.98 \\
\hline Treated with penicillamine & $13(54)$ & $42(66)$ & 0.46 \\
\hline Diffuse SSc & $7(29)$ & $19(39)$ & 0.83 \\
\hline Arthritis & $4(17)$ & $3(5)$ & 0.08 \\
\hline SSc joint deformity & $19(79)$ & $42(66)$ & 0.33 \\
\hline Pitting scars & $17(71)$ & $42(66)$ & 0.83 \\
\hline Calcinosis & $12(50)$ & $29(45)$ & 0.88 \\
\hline Distal osteolysis & $6(25)$ & $13(20)$ & 0.85 \\
\hline Pulmonary fibrosis & $11(46)$ & $16(25)$ & 0.10 \\
\hline Restrictive ventilatory pattern & $7(29)$ & $10(16)$ & 0.22 \\
\hline Obstructive ventilatory pattern & $7(29)$ & $4(6.3)$ & 0.008 \\
\hline Isolated reduction of DLco & $8(33)$ & $29(45)$ & 0.44 \\
\hline Continuous variables & Median (quartiles) & Median (quartiles) & $\mathrm{p}^{\dagger}$ \\
\hline Age (years) & $66(54-71)$ & $56(44-67)$ & 0.01 \\
\hline Disease duration (years) & $7.8(3.4-17)$ & $6.9(3.9-15)$ & 0.86 \\
\hline Vital capacity (\% of expected) & $92(70-105)$ & $102(86-117)$ & 0.01 \\
\hline $\mathrm{FEV}_{1} / \mathrm{NC}(\%)$ & $81(69-85)$ & $82(77-87)$ & 0.11 \\
\hline Diffusing capacity (\% of expected) & $58(52-73)$ & $74(62-86)$ & 0.003 \\
\hline
\end{tabular}

*Fisher's exact test. †Mann-Whitney test.

Immunohistochemical analysis for YKL-40 protein expression in a lung biopsy with inflammation and severe fibrosis from a patient who died of SSc showed YKL-40 expression in macrophages and leucocytes in areas with inflammation (data not shown).

Twenty-two (25\%) patients died during follow-up, and 11 of these had elevated serum YKL-40. Nine patients died of causes related to SSc [pulmonary fibrosis (six), renal crisis (one), and malabsorption (two)], and six of these had elevated serum YKL-40. The patients who died had higher serum YKL-40 (median $264 \mu \mathrm{g} / \mathrm{L}$, quartiles: $188-352 \mu \mathrm{g} / \mathrm{L}$ ) than the surviving patients (median $144 \mu \mathrm{g} / \mathrm{L}$, quartiles: 96-244 $\mu \mathrm{g} / \mathrm{L}, \mathrm{p}<0.001)$. Patients with high serum YKL-40 had shorter survival from the time of the 
blood sample than patients with normal serum YKL$40(p=0.004)$ (Figure 1). When controlling for age, reduced vital capacity, and reduced diffusing capacity, patients with increased serum YKL-40 still had the poorest survival, although this was not statistically significant $(\mathrm{p}=0.1)$.

\section{Discussion}

We found elevated serum YKL-40 in 27\% of patients with SSc, similar to other reports (13). SSc patients with various signs of pulmonary involvement had elevated serum YKL-40, and this was associated with features of obstructive lung disease and decreased vital and diffusing pulmonary capacity. The cellular abnormalities in SSc lungs often result in complex alterations of the lung function. Interstitial fibrosis, vascular lesions including fibrous thickening of the intima, media hypertrophy, and perivascular fibrosis may lead to a restrictive lung function pattern and/or impairment of the diffusing capacity. High serum YKL-40 was related to reduced DLco, possibly caused by interstitial and perivascular fibrosis. Interestingly, the present study shows that SSc patients with elevated serum YKL-40 had shorter survival than patients with normal serum YKL-40 and died more often due to extensive interstitial or vascular fibrosing processes (e.g. pulmonary fibrosis, $\mathrm{SSc}$ renal crisis). However, this association is partly explained by age, reduced vital capacity, and reduced diffusing capacity.

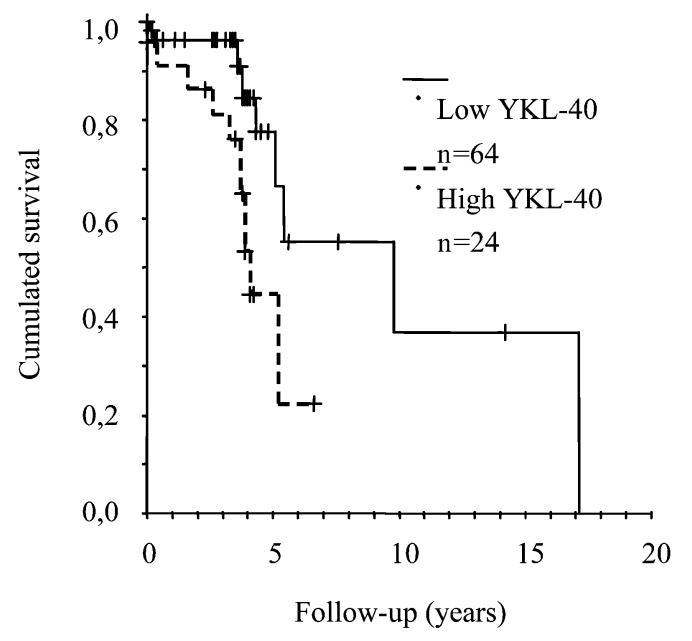

Figure 1. The impact of serum YKL-40 on overall survival of patients with systemic sclerosis. The patients were grouped by a high versus normal (age-adjusted) serum YKL-40. The strata are: 1 (-) patient having normal serum YKL-40 (i.e. $\leqslant 275 \mu \mathrm{g} / \mathrm{L}$ ); 2 (---) patients having elevated serum YKL-40 (i.e. $>275 \mu \mathrm{g} / \mathrm{L}$ ). The cut-off limit used for the determination of normal versus high serum YKL-40 was the 95th confidence limit of serum YKL-40 in healthy age-matched subjects. The p-value shown is for the log rank test for equality of strata.
Immunohistochemical analysis of a lung biopsy from an SSc patient with lung inflammation and fibrosis showed YKL-40 protein expression in macrophages and neutrophils in areas with inflammation. YKL-40 is a growth factor for fibroblasts (7), but it is not known whether YKL-40 plays a role in the pathogenesis of pulmonary fibrosis in patients with SSc. YKL-40 may actively take part in inflammation and tissue remodelling processes and has a role in pathological conditions leading to organ fibrosis. YKL-40 was one of the most differentially expressed genes in liver tissue with hepatitis C cirrhosis (15) and serum YKL-40 was elevated in most patients with moderate to severe liver fibrosis and cirrhosis, independently of disease aetiology (11) associated with shorter survival of patients with alcoholic liver disease compared to patients with normal serum YKL-40.

The precise role of YKL-40 in the pathogenesis of pulmonary fibrosis in SSc is still unknown and large prospective studies of patients with SSc are needed to determine any prognostic significance of high serum YKL-40.

\section{Acknowledgements}

We appreciate the excellent technical assistance by the deceased Inger Aakard, Department of Rheumatology, Hvidovre Hospital, Denmark. Sussi Forchammer, Institute of Medical Anatomy, Section A, The Panum Institute, University of Copenhagen, Denmark is thanked for the immunohistochemical analysis of the lung biopsy.

\section{References}

1. Hakala BE, White C, Recklies AD. Human cartilage gp-39, a major secretory product of articular chondrocytes and synovial cells, is a mammalian member of a chitinase protein family. J Biol Chem 1993;268:5803-10.

2. Rehli M, Krause SW, Andreesen R. Molecular characterization of the gene for human cartilage gp-39 (CHI3L1), a member of the chitinase protein family and marker for late stages of macrophage differentiation. Genomics 1997;43:221-5.

3. Houston DR, Recklies AD, Krupa JC, van Aalten DMF. Structure and ligand-induced conformational change of the $39-\mathrm{kDa}$ glycoprotein from human articular chondrocytes. J Biol Chem 2003;278:30206-12.

4. Renkema GH, Boot RG, Au FL, Donker-Koopman WE, Strijland A, Muijsers AO, et al. Chitotriosidase, a chitinase, and the 39-kDa human cartilage glycoprotein, a chitin-binding lectin, are homologues of family 18 glycosyl hydrolases secreted by human macrophages. Eur J Biochem 1998;251:504-9.

5. Boot RG, van Achterberg TAE, van Aken BE, Renkema GH, Jacobs MJHM, Aerts JMFG, et al. Strong induction of members of the chitinase family of proteins in atherosclerosis. Chitotriosidase and human cartilage gp-39 expressed in lesion macrophages. Arterioscler Thromb Vasc Biol 1999;19:687-94.

6. Volck B, Price PA, Johansen JS, Sørensen O, Benfield TL, Nielsen HJ, et al. YKL-40, a mammalian member of the chitinase family, is a matrix protein of specific granules in human neutrophils. Proc Assoc Am Physicians 1998;110: $351-60$. 
7. Recklies AD, White $\mathrm{C}$, Ling $\mathrm{H}$. The chitinase 3-like protein human cartilage 39 (HC-gp39) stimulates proliferation of human connective-tissue cells and activates both extracellular signal-regulated kinase and protein kinase B-mediated signalling pathways. Biochem J 2002;365:119-26.

8. De Ceuninck F, Gaufillier S, Bonnaud A, Sabatini M, Lesur C, Pastoureau P. YKL-40 (cartilage gp-39) induces proliferative events in cultured chondrocytes and synoviocytes and increases glycosaminoglycan synthesis in chondrocytes. Biochem Biophys Res Commun 2001;285:926-31.

9. Nishikawa KC, Millis AJT. Gp38k (CHI3L1) is a novel adhesion and migration factor for vascular cells. Exp Cell Res 2003;287:79-87.

10. Johansen JS, Christoffersen P, Møller S, Price PA, Henriksen JH, Garbarsch C, et al. Serum YKL-40 is increased in patients with hepatic fibrosis. J Hepatol 2000;32: 911-20.
11. Jensen BV, Johansen JS, Price PA. High levels of serum HER2/neu and YKL-40 independently reflect aggressiveness of metastatic breast cancer. Clin Cancer Res 2003;9:501-12.

12. Takehara K. Pathogenesis of systemic sclerosis. J Rheumatol 2003;30:755-9.

13. Montagna GL, D'Angelo S, Valentini G. Cross-sectional evaluation of YKL-40 serum concentration in patients with systemic sclerosis. Relationship with clinical and serological aspects of disease. J Rheumatol 2003;30:2147-51.

14. Jacobsen S, Halberg $\mathrm{P}$, Ullman S, Høier-Madsen $\mathrm{M}$, Petersen J, Mortensen J, et al. A longitudinal study of pulmonary function in Danish patients with systemic sclerosis. Clin Rheum 1997;16:384-90.

15. Shackel NA, McGuinness PH, Abbott CA, Gorrell MD, McCaughan GW. Novel differential gene expression in human cirrhosis detected by suppression subtractive hybridisation. Hepatology 2003;38:577-88. 